\title{
NEUROCIÊNCIA E DESIGN BIOFÍLICO APLICADOS AO URBANISMO: A RELAÇÃO ENTRE A CIDADE E A SAÚdE DO USUÁRIO
}

\section{ARTIGO DE REVISÃO}

ALMEIDA, Karla Carvalho de ${ }^{1}$, SOUZA, Rosiane de Oliveira ${ }^{2}$, COSTA, Naiara Vilela ${ }^{3}$ ALMEIDA, Karla Carvalho de. SOUZA, Rosiane de Oliveira. COSTA, Naiara Vilela. Neurociência e design biofílico aplicados ao urbanismo: a relação entre a cidade e a saúde do usuário. Revista Científica Multidisciplinar Núcleo do Conhecimento. Ano. 07, Ed. 01, Vol. 02, pp. 65-79. Janeiro de 2022. ISSN: 24480959, Link de acesso: https://www.nucleodoconhecimento.com.br/arquitetura/neurociencia-e-design, DOI: 10.32749/nucleodoconhecimento.com.br/arquitetura/neurociencia-e-design

\section{RESUMO}

A maior parte da população atual vive em área urbana, e a rotina nas cidades comumente acarreta problemas de saúde física, mental e emocional, como: estresse, ansiedade, depressão, obesidade, entre outros. Desde que o ser humano passou a habitar a zona urbana, faz-se essencial planejar espaços públicos de acordo com as suas necessidades psicológicas, físicas e sociais. Neste contexto, o presente artigo, tem como questão norteadora: Qual é o papel do neurourbanismo e, consequentemente, da biofilia no planejamento de cidades promotoras de saúde e bem-estar? O objetivo do trabalho é discorrer sobre os princípios teóricos da neurociência aplicada ao urbanismo, os efeitos de elementos urbanos no cérebro do

\footnotetext{
${ }^{1}$ Pós-graduação em Arquitetura da Paisagem pela Pontifícia Universidade Católica de Minas Gerais (PUCMinas) e graduação em Arquitetura e Urbanismo pela Universidade Federal de Juiz de Fora (UFJF). ORCID: 0000-0003-2425-4382.

${ }^{2}$ Graduação em Arquitetura e Urbanismo pela Universidade Federal de Juiz de Fora (UFJF). ORCID: 0000-00015760-8189.

${ }^{3}$ Graduação em Arquitetura e Urbanismo pela Universidade Federal de Juiz de Fora (UFJF). ORCID: 0000-0001$8382-7780$
}

RC: 104824

Disponível em: https://www.nucleodoconhecimento.com.br/arquitetura/neurocienciae-design 
habitante, a estratégia da biofilia como solução urbana e suas diversas formas de aplicação, a fim de promover o bem-estar e a saúde dos usuários. Como metodologia, foi desenvolvida a revisão bibliográfica de conceitos relacionados à neurociência, urbanismo, arquitetura, biofilia e suas aplicações; e a análise de estudos de caso sobre a experiência de indivíduos e o ambiente que os cerca. Como resultado do trabalho, são destacados os efeitos das cidades e da biofilia no cérebro humano, além de apresentar diretrizes a se considerar em projetos urbanos de acordo com o design biofílico e embasados na neurociência. Concluindo, então, na demonstração da importância de sua aplicação para efeitos satisfatórios de promoção de saúde e bem-estar em áreas urbanas.

Palavras-chave: neurociência, urbanismo, neurourbanismo, neuroarquitetura, biofilia.

\section{INTRODUÇÃO}

O ser humano cria e modifica não apenas seus espaços, mas também a sua mente: "Todo homem pode, se assim se propuser, ser o escultor de seu próprio cérebro" (RAMÓN e CAJAL, 2004). Neurourbanismo e Neuroarquitetura são disciplinas resultantes da mistura entre Neurociência e Urbanismo ou Arquitetura, respectivamente. Consiste no estudo da relação entre as pessoas e o ambiente, especialmente no que concerne às mudanças psicológicas, físicas e emocionais relacionadas à estadia de longa e curta duração em determinados espaços. $O$ neurourbanismo, por sua vez, foca na aliança entre as questões urbanas e a saúde, em como a vida na cidade afeta o cérebro e as emoções do usuário, a fim de promover espaços urbanos com estratégias que causem o bem-estar e qualidade de vida (PAIVA, 2018).

É comum arquitetos e urbanistas terem consciência do impacto de seus projetos nas vidas dos usuários do local. O psicólogo Kurt Lewin (1890-1947) previu essa relação entre ambiente e usuário através da equação $C=f\left(P^{*} M\right)$, em que o Comportamento (C) é a função entre a Pessoa (P) e o seu Meio Ambiente (M). Dessa forma, um indivíduo com vivências e genéticas distintas é influenciado pelo seu meio, não 
apenas social, mas também o físico - assumindo a responsabilidade da arquitetura no resultado de seu comportamento (PAIVA, 2018).

De acordo com a neurociência, a habilidade de processar informações de forma consciente é menor em cerca de $99 \%$ quando comparada à forma inconsciente. Esse dado mostra que os estímulos ambientais influenciam seus usuários, majoritariamente, de forma inconsciente e involuntária (PAIVA, 2018).

As cidades, por sua vez, formam o cenário em que a maioria das pessoas passa toda a sua vida. O cotidiano urbano traz benefícios econômicos e práticos à população, mas a vida na cidade pode apresentar desafios à saúde de seus habitantes, como obesidade e desequilíbrio mental e emocional. A rotina urbana comumente acarreta na sensação de solidão, estresse, poluição visual, olfativa, respiratória e sonora - com frequentes sons em decibéis altos como a sirene de uma ambulância por exemplo (JACOBS, 2011). As previsões são de que, até 2050, pelo menos $70 \%$ da população mundial viverá em áreas urbanas. Desta forma, mais pessoas estarão expostas ao estresse diário e as cidades ficarão ainda mais cheias. É preciso entender como e quanto isso afeta as pessoas, a fim de estabelecer medidas que melhorem as condições de quem mora nas grandes cidades (ONU, 2019).

Diante dessa realidade, qual é o papel do neurourbanismo e, consequentemente, da biofilia no planejamento de cidades promotoras de saúde e bem-estar? O objetivo do trabalho é discorrer sobre alguns dos principais fundamentos teóricos da neurociência aplicada ao urbanismo, os efeitos de elementos urbanos no cérebro do habitante, e o princípio da biofilia como solução urbana e suas diversas formas de aplicação, a fim de promover o bem-estar e a saúde dos usuários.

\section{METODOLOGIA}

A metodologia empregada neste artigo aborda: (i) pesquisa bibliográfica sobre o tema neurourbanismo; (ii) estudos de caso sobre a neurociência aplicada a 
elementos do urbanismo, sobre psicologia e a sua influência em situações ligadas ao cotidiano; (iii) revisão bibliográfica sobre questões ligadas à medicina, bem como das referências sobre as áreas voltadas ao tema, que auxiliaram no levantamento de dados sobre a importância da vivência na natureza a todas as pessoas; (iv) levantamento empírico dos aspectos urbanos que impactam positiva e negativamente no modo de vida, a fim de se estabelecer um diagnóstico.

O artigo foi estruturado de forma a apontar a importância da aplicação da neurociência ao urbanismo, com justificativas e estratégias de execução. Inicialmente, tem-se o referencial teórico, onde é realizada uma breve análise dos principais campos a serem estudados: a relação entre arquitetura, urbanismo e neurociência; e a conceituação de biofilia e de seus serviços ecossistêmicos.

Em seguida, as seções abordam as pesquisas relacionadas ao efeito do ambiente construído na saúde do usuário, a fim de esclarecer, de acordo com o embasamento teórico, sobre como acontece a absorção de informações pelo cérebro e seus efeitos de curto e longo prazo. Logo após, são abordados os efeitos da biofilia na saúde física e mental, respectivamente. Por fim, são expostas as diretrizes projetuais baseadas no design biofílico, e suas diversas formas de implementação.

\section{FUNDAMENTAÇÃO TEÓRICA}

A arquitetura e o urbanismo afetam diretamente a plasticidade cerebral - capacidade de mudança física do cérebro, principalmente em relação às conexões entre neurônios, de acordo com os estímulos (ou falta deles) que recebe do externo. Ambientes carentes em estímulos físico-sensoriais podem causar perda de volume em algumas áreas do cérebro, resultante principalmente da diminuição e do enfraquecimento das sinapses. Geralmente, quanto mais tempo um usuário passa em um determinado ambiente, por mais tempo ele afetará o seu cérebro. Sendo assim, _habitações, locais de trabalho e cidades são exemplos de espaços que tendem a ser ocupados por muitas horas frequentemente ao longo de anos e, 
portanto, têm maior potencial de gerar efeitos mais duradouros em seus usuários (JEDON e PAIVA, 2019).

Um dos aspectos a se considerar para um ambiente construído saudável consiste na Biofilia. Trata-se do estudo de como a natureza pode influenciar no cérebro e no corpo de forma geral. A Biofilia defende que, quanto mais contato com elementos da natureza no dia a dia, maior o índice de imunidade, por exemplo, o indivíduo apresenta (SALINGAROS, 2015).

A natureza fornece diversos serviços ecossistêmicos ao local em que está inserida. Por definição, serviços ecossistêmicos consistem em componentes da natureza apreciados, consumidos ou utilizados direta ou indiretamente para produzir o bemestar humano (BOYD; BANZHAF, 2007). Trata-se de benefícios proporcionados pela natureza como, por exemplo, água potável produzida pelo regime de chuvas controlado pelas florestas nativas, umidade e temperatura regulados, qualidade saudável do ar, parques e praças públicas de recreação, estabilização do solo pelas raízes arbóreas, dentre diversos outros.

Serviços ecossistêmicos podem ser gerados por fragmentos naturais, como massas florestais nativas remanescentes, árvores urbanas, parques, praças, wetlands construídos, ou lajes vegetalizadas, por exemplo (BEATLEY e MCDONALD, 2021).

Nos itens seguintes, é discutido como a biofilia pode impactar a saúde física e mental, respectivamente.

\section{ABSORÇÃO DE INFORMAÇÕES E SEUS EFEITOS NO CÉREBRO}

Uma maneira simplificada de analisar o cérebro humano, na neurociência, consiste em classificá-lo em dois sistemas: sistema I - pensamento rápido e automático - e sistema II - pensamento lento e elaborado (KAHNEMAN, 2011).

O sistema I consiste em um nível inconsciente de absorção de informação: é rápido, automático, intuitivo, impulsivo e emocional. É responsável por controlar as funções 
que mantêm o corpo em segurança. O sistema II é consciente, lento, racional, sistemático, analítico e reflexivo. Todas as ações e reações humanas são guiadas por esses dois sistemas, que comportam proporções diferentes no poder de decisão. O sistema II toma decisões novas, por exemplo, até que elas se tornem automáticas e, portanto, comandadas pelo sistema I - que exerce a maior parte do controle nessa disputa (PAIVA, 2018).

Essa vantagem emocional ocorre porque o sistema I precisa de menos energia para funcionar do que o sistema II. O cérebro humano, por sua vez, é programado para poupar energia, fazendo com que a maioria das decisões sejam tomadas por este sistema emocional.

Diante desses fatos, é importante que os arquitetos e urbanistas tenham consciência para qual destes dois sistemas estão projetando.

Através de tais estímulos, o cérebro pode lidar com efeitos de curta ou de longa duração. Efeito de curto prazo ocorre como uma consequência à interação com determinado ambiente, que não tem longa duração. Acontece para que o indivíduo se adapte àquele espaço enquanto estiver habitando-o. Tais mudanças (que consistem normalmente em alterações hormonais, de capacidade de memorização, batimentos cardíacos, pressão sanguínea, temperatura corporal ou tensão muscular) podem acabar poucas horas depois que a pessoa deixa o local. Por exemplo, uma pessoa em uma situação real de perigo pode sentir medo e aceleração dos batimentos cardíacos, para que ela se empenhe em sair do local o mais rápido possível. Ao chegar em casa, geralmente o indivíduo se acalma (JEDON e PAIVA, 2019).

Os efeitos de longo prazo, por sua vez, geralmente necessitam de longa exposição a determinado ambiente causador dessa consequência; ao passo que também exigem mais tempo para terem seus efeitos eliminados ou minimizados. Por exemplo, passar um dia em casa pode ajudar a diminuir os níveis de estresse e ajudar a relaxar - efeitos de curto prazo. Porém, um aposentado que está há anos em casa e raramente vai a outros locais pode apresentar mudanças plásticas em seu cérebro, 
perdendo eficiência e rapidez com o tempo - efeitos de longo prazo (JEDON e PAIVA, 2019).

É importante ressaltar, porém, que o estresse não é completamente nocivo ao cérebro: é ele o responsável por emitir a reação da fuga em determinadas situações de perigo. Antigamente, por exemplo, era ele o responsável por fazer os ancestrais fugirem de predadores, $O$ estresse agudo (momentâneo) produz a energia necessária para ter força e velocidade para escapar do perigo (PAIVA, 2019).

$\mathrm{Na}$ contemporaneidade, a rotina adotada pela maioria dos seres humanos, gera um tipo de estresse diferente: o estresse crônico. Que pode ser exemplificado em certas situações, tais como o receio de assaltos, a corrida contra o tempo para conseguir realizar todas as tarefas, ambas são situações geradoras do estresse crônico (PAIVA, 2019).

O estresse agudo, acontece de maneira pontual e intensa, com a liberação de um hormônio chamado cortisol, que além de promover papel importante no auxílio do estado de fuga, age como anti-inflamatório natural, auxiliando a controlar o metabolismo e aumentar a sensação de energia e a memória (PAIVA, 2019).

Por sua vez, o estresse crônico acontece em doses menores, porém de forma contínua e isto torna-se prejudicial à saúde, visto que pode causar problemas como a supressão do sistema imunológico e da memória, além de desencadear uma síndrome metabólica e perda de mineral nos ossos, ou seja, efeitos contrários devido à dosagem contínua do hormônio. Devido aos problemas cotidianos atuais, torna-se desafiador transitar nas vias sem nenhum receio, ou ter uma condição de vida estável, de forma que não seja necessário possuir dois ou mais empregos (PAIVA, 2019).

É importante ressaltar que cada pessoa possui um metabolismo diferente, a depender de sua genética, historicidade física e emocional, ambiente em que vive, entre outras características individuais do ser, então cada indivíduo pode sofrer formas diversificadas da dosagem contínua de cortisol, mas é preciso salientar que o

RC: 104824

Disponível em: https://www.nucleodoconhecimento.com.br/arquitetura/neurocienciae-design 
corpo humano e, principalmente, o cérebro não são capazes de sobreviver sendo continuamente afetados pelo estresse crônico (PAIVA, 2019).

\section{EFEITOS DAS CIDADES NA SAÚDE DO USUÁRIO}

A interação com o meio urbano pode impactar positiva ou negativamente no cérebro humano, por meio de estratégias de planejamento, e isto vai depender de como tal meio foi utilizado. As características naturais sempre são meios de interagir positivamente com o indivíduo, mas com a evolução das tecnologias dos materiais, no contexto da arquitetura e do urbanismo, foram surgindo novos valores estéticos que transformaram grande parte das edificações em escalas métricas que seguem um valor puramente econômico, o que por consequência se fez perder a característica natural (PAIVA, 2019).

Esta perda acontece também na escala urbana: os arranha-céus delimitam o espaço visível, quebram paisagens naturais, distanciam o ser humano da natureza. As distâncias entre os lugares se tornam longas a ponto de ser inviável os meios de transporte não poluentes, como bicicleta e caminhada, bem como as vias priorizam veículos e a natureza perde espaço para o vazio do ir e vir cotidiano, da rotina em estresse crônico (PAIVA, 2019).

Jacobs (2011) destaca a importância de a cidade, por si só, ser um atrativo e de provocar a sensação de pertencimento, ser um território conhecido, lugar onde é possível estar por longos períodos, de maneira prazerosa e relaxada. O meio urbano precisa, então, ser um local de baixa chance de surgimento de qualquer tipo de ameaça. Esse pertencimento é o que gera no ser humano a vontade de cuidar ( manter a cidade em bom estado de conservação).

Atualmente, porém, a cidade passou a ser uma extensão de um corpo desconhecido, devido a edificações sempre iguais, meramente criadas pelo atrativo econômico. Isso ocasiona desprendimento regional, pois não é possível criar a conexão cerebral para associar determinada tipologia de edificação a um local 
definido na região, gerando perda de referência do próprio habitante da cidade. A partir do desconhecido, não é possível criar uma ligação, uma conexão ao pertencimento, o que explica a falta de cuidados em diversos locais ( $\mathrm{LYNCH}, 2008)$.

O neurourbanismo entra como fator estratégico contrapondo à concepção massiva cinzenta de cidade contemporânea. Com estratégias e técnicas de planejamento, investimento e mudanças de hábitos, como forma de trazer de volta o senso de pertencimento, a qualidade de vida e a segurança aos cidadãos, cria-se uma conexão positiva com a cidade, a fim de que o usuário possa usufruir de melhor qualidade de vida e sinta-se responsável pelos cuidados de manutenção e de preservação dos espaços públicos.

\section{EFEITOS DA BIOFILIA NA SAÚDE FÍSICA}

Uma das formas em que parques e outros recursos naturais afetam a saúde é através do incentivo ao exercício físico proporcionado pelas áreas de recreação (BEATLEY e MCDONALD, 2021). Um estudo em Los Angeles, por exemplo, feito por Wolch et al. (2011), rastreou a saúde de mais de 3000 crianças por duas décadas. As conclusões foram de que crianças que vivem a até 500 metros de distância de parques apresentam menor índice de massa corporal (IMC) e melhor resultados de saúde. Em uma maior escala, Ewing et al. (2003) revisou todos os condados dos Estados Unidos e correlacionou um índice de expansão urbana com dados de caminhada e medidas de IMC. Os resultados mostraram que os condados com mais expansão tinham menos atividade de caminhada e maior IMC. Dessa forma, aumentar o acesso aos parques agora é uma das estratégias listadas no guia dos Centros de Controle de Doenças - linhas para aumentar a atividade física em uma comunidade (CDC, 2011).

Tão importante quanto a proximidade em relação a parques é garantir que haja outros recursos que os residentes desejem utilizar, como trilhas, playgrounds, praças, entre outros. Uma das estratégias listadas pela CDC consiste em proporcionar ruas caminháveis. Aumentar a cobertura do dossel, garantir segurança 
e facilidade de movimentação, proporcionar temperatura e umidade adequadas através da arborização são algumas técnicas para aumentar a caminhabilidade.

Além disso, a infraestrutura verde pode ajudar a diminuir a temperatura do ar. Árvores e outras vegetações podem sombrear superfícies impermeáveis e impedir que elas se aqueçam. As plantas também transpiram ao longo de seu crescimento, refrescando o ar. Em dias quentes de verão, tais recursos trazem benefícios significativos à saúde. Na maioria dos anos, as ondas de calor são a maior fonte de mortalidade relacionada ao clima em todo o mundo, matando em média 12.000 pessoas (MCMICHAEL et al., 2004). Um estudo realizado por Anderson e Bell (2011) descobriu que cada aumento de $0,6^{\circ} \mathrm{C}\left(1^{\circ} \mathrm{F}\right)$ na temperatura do ar aumentava o risco relativo de mortalidade nas cidades dos EUA em $2,5 \%$.

A limpeza do ar é outro benefício proporcionado pela vegetação urbana. As folhas servem como superfície para as partículas secas se depositarem, assim como a poeira se acumula nos móveis de uma casa. Isto é importante porque essas partículas são uma grande ameaça à saúde pública: ao ar livre são inaladas para os pulmões, causando sérios impactos à saúde, como doenças cardiovasculares e asma - sendo a causa de morte de mais de três milhões de pessoas por ano. As árvores também podem modular as concentrações de ozônio e outros poluentes atmosféricos (BEATLEY e MCDONALD, 2021).

\section{EFEITOS DA BIOFILIA NA SAÚDE MENTAL}

Interagir com elementos naturais é importante não só para a saúde física, mas também para a saúde mental. A redução de estresse pelo contato com a natureza foi provada por meio de relatos (STIGSDOTTER et al., 2010) e por meio da redução do nível de cortisol (THOMPSON et al., 2012). Esse efeito é observado, também, em ambientes de trabalho que proporcionam contato com plantas, apresentando menor estresse, menos reclamações relacionadas à saúde e menor índice de faltas (BJORNSTAD et al., 2016).

RC: 104824

Disponível em: https://www.nucleodoconhecimento.com.br/arquitetura/neurociencia$\underline{\text { e-design }}$ 
Estudos demonstram que exposição à natureza está relacionada com a melhoria na saúde mental autorrelatada e com a redução do índice de depressão e de esquizofrenia (SUNDQUIST et al., 2004).

Isso ocorre porque áreas naturais, entre outros fatores, têm menos estressores do que ambientes construídos de forma geral, como poluição sonora, por exemplo. Plantas são ótimas geradoras de conforto acústico, diminuindo esse tipo de estresse (BEATLEY e MCDONALD, 2021).

O relaxamento causado pelo contato com a natureza, porém, tem mais causas do que apenas a redução de estressores. Uma das explicações consiste na teoria da restauração da atenção (TRA), proposta pela primeira vez por Kaplan e Stephen Kaplan (1989). Esta hipótese argumenta que a vida moderna requer muita atenção direcionada, como por exemplo monitorar os sons vindos de celulares, responder a demandas de trabalho, prezar pela segurança nas ruas, entre diversos outros. Toda essa atenção direcionada é cansativa para as psiques, que não evoluíram para tal solicitação constante.

O cenário natural, por sua vez, requer atenção menos direcionada, gerando uma pausa restauradora. Alguns estudos sugeriram que os ambientes naturais chamam atenção de uma maneira agradável e sem exigir esforço. Este efeito foi confirmado com experiências em que os movimentos dos olhos dos usuários do local foram analisados (BERTO, 2005). Após passar esse intervalo em um ambiente natural, a capacidade de atenção direcionada e a memória de curto prazo são aumentadas novamente (BERMAN et al., 2008).

Outra hipótese consiste na teoria da redução de estresse (TRE), que defende que o efeito calmante se dá pelo fato de que seres humanos evoluíram como espécie para esse tipo de ambiente, tendo passado a maior parte da história evolutiva imersos na natureza. Uma evidência para esse resultado se dá pela diminuição de cortisol após uma caminhada na floresta (ULRICH, 1983). 
Imagem 01: Períodos de evolução da espécie humana e seus cenários.

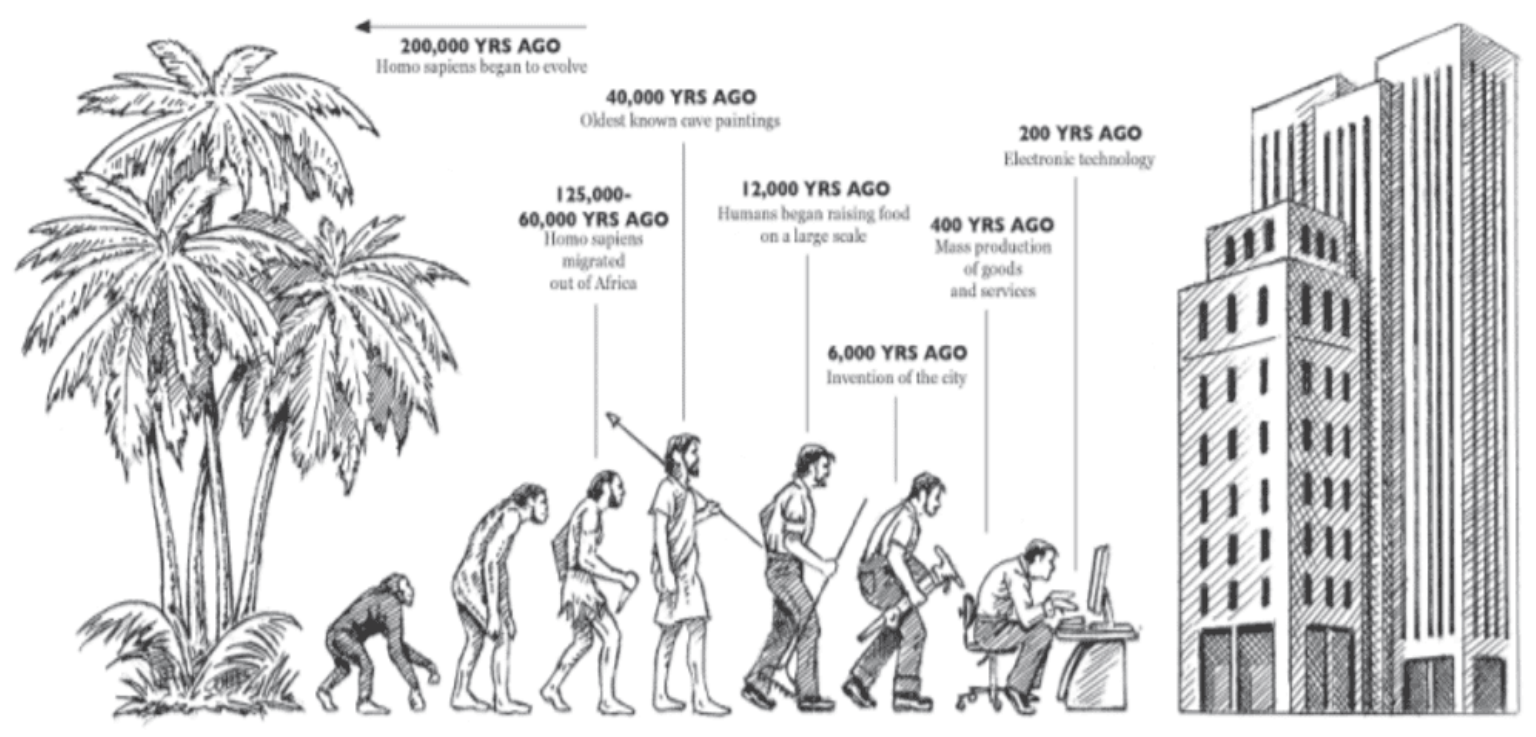

Fonte: KELLERT, 2018.

Essa teoria está diretamente relacionada à premissa da biofilia que defende que, como espécie, o ser humano tem uma conexão inata com a natureza: é capaz de ser mais feliz e saudável quando a natureza está presente onde vive e trabalha. Designers e planejadores biofílicos argumentam que a integração da natureza ao ambiente construído é um passo importante em direção às cidades do futuro que são edificantes, restauradoras, esteticamente agradáveis e projetadas em torno de um senso de conexão e de admiração com o mundo natural (BEATLEY e MCDONALD, 2021).

\section{BIOFILIA APLICADA}

Estratégias de design podem aumentar significativamente o efeito da aplicação de biofilia em ambientes construídos. Elas são divididas principalmente em três categorias: contato direto e contato indireto com a natureza, e experiência de espaço. Esses atributos refletem o quão bem-sucedidos ambientes humanos (aqueles que promovem boa saúde e maior produtividade) dependem da criação de

RC: 104824

Disponível em: https://www.nucleodoconhecimento.com.br/arquitetura/neurocienciae-design 
habitats de partes complementares e conectadas que compreendem a totalidade ecológica (KELLERT, 2018).

A primeira categoria consiste em interagir com recursos naturais básicos como luz solar, ar, água, plantas, animais, vista de paisagens naturais, clima e fogo (KELLERT, 2018). Já a segunda, por sua vez, se baseia em imagens, materiais, texturas, cores, formas, detalhes, pátinas, geometrias, simulação de iluminação e ventilação naturais e biomimetismo - tudo isso transformado do seu estado natural. $\mathrm{Na}$ prática, inclui fotografias; texturas de madeira, pedra, couro e metal; cores comumente presentes na natureza, como verde e tons terrosos; formas orgânicas distribuídas no espaço, como móveis arredondados e caminhos tortuosos; entre outros elementos que remetem à natureza (KELLERT, 2018).

O terceiro elemento básico do design biofílico é a experiência de espaço e lugar. Atributos associados à experiência de espaço e lugar incluem perspectiva e refúgio (discernimento de longas distâncias de um espaço protegido e seguro), complexidade organizada (equilíbrio de detalhes e diversidade), espaços de transição (ligando ambientes internos e externos), mobilidade, conexões ecológicas e culturais com o local e a integração das partes com o todo (KELLERT, 2018).

\section{CONSIDERAÇÕES FINAIS}

De acordo com o embasamento teórico, foi possível perceber os efeitos nocivos que as cidades contemporâneas vêm causando em seus moradores, devido a comumente não contemplarem os conceitos básicos de neurociência aplicada ao urbanismo.

A biofilia, por sua vez, mostrou-se como potencialmente benéfica ao ser humano por, historicamente, ter sido seu habitat por um período de tempo significativamente maior do que os centros urbanos. É notável, de acordo com a pesquisa, as vantagens neurológicas de curto e longo prazo, psicológicas e físicas que o contato (direto ou indireto) com elementos naturais é capaz de proporcionar ao usuário. 
A aplicação da biofilia se mostrou possível em inúmeras variações, desde o aproveitamento de elementos básicos como a iluminação natural, o ar e a água, até a utilização de materiais que remetem esteticamente aos naturais, trazendo a sensação de relaxamento ao ser humano.

Por fim, retomando a questão norteadora: Qual é o papel do neurourbanismo e, consequentemente, da biofilia no planejamento de cidades promotoras de saúde e bem-estar? O trabalho destacou o papel de diversos princípios da neurociência no auxílio ao planejamento de cidades promotoras de saúde e bem estar, auxiliando na memória, diminuindo a ansiedade e o estresse, promovendo atividade física através da caminhabilidade, e mantendo o cérebro saudável. A biofilia, por sua vez, se mostrou como elemento fundamental - baseado na neurociência aplicada ao urbanismo - para o alcance de tais objetivos.

\section{REFERÊNCIAS}

ANDERSON, G. Brooke; BELL, Michelle L. Heat waves in the United States: Mortality risk during heat waves and effect modification by heat wave characteristics in 43 US communities. Environmental Health Perspectives 119 (2): 210, 2011.

BEATLEY, Tim; MCDONALD, Robert. Biophilic Cities For An Urban Century: Why nature is essential for the success of the cities. Palgrave Macmillan, 2021.

BERMAN, Marc G.; JONIDES, Jhon; KAPLAN, Stephen. The cognitive benefits of interacting with nature. Psychological Science 19: 1207-1212, 2008.

BERTO, Rita. Exposure to restorative environments helps restore attentional capacity. Journal of Environmental Psychology 25 (3): 249-259, 2005.

BJORNSTAD, Siv. et al. Nature contact and organizational support during office working hours: Benefits relating to stress reduction, subjective health complaints, and sick leave. ResearchGate, 2014. Disponível em: $<$ https://www.researchgate.net/publication/287483079_Nature_contact_and_organiz 
ational_support_during_office_working_hours_Benefits_relating_to_stress_reduction _subjective_health_complaints_and_sick_leave>. Acesso em: 06 de dez. de 2021.

BOYD, James; BANZHAF, Spencer. What are ecosystem services? The need for standardized environmental accounting units. Ecological EconomicsDiscussion Paper, 2006.

CDC. Strategies to prevent obesity and other chronic diseases: The CDC guide to strategies to increase physical activity in the community. Atlanta: U.S. Department of Health and Human Services, Centers for Disease Control and Prevention, 2011. Disponível em: <https://www.cdc.gov/obesity/downloads/pa_2011_web.pdf>. Acesso em: 06 de dez. de 2021.

EWING, Reid. et al. Relationship between urban sprawl and physical activity, obesity, and morbidity. National Library of Medicine, 2003. Disponível em: <https://pubmed.ncbi.nlm.nih.gov/13677962/>. Acesso em: 06 de dez. de 2021.

JACOBS, Jane. Morte e vida das grandes cidades. São Paulo: WMF Martins Fontes, $3^{\circ}$ edição, 2011.

JEDON, Richard; PAIVA, Andrea. Short and long-term effects of architecture on the brain: toward theoretical formalization. Science Direct. Frontiers of Architectural Research, 2019. Volume 8, Issue 4, Dezembro de 2019 , páginas 564-571. Disponível em: <https://www.sciencedirect.com/science/article/pii/S2095263519300585>. Acesso em: 06 de dez. de 2021.

KAHNEMAN, Daniel. Thinking: Fast and Slow. New York: Farrar, Straus and Giroux, 2011.

KAPLAN, Rachel; KAPLAN, Stephen. The experience of nature: A psychological perspective. New York: Cambridge University Press, 1989. Disponível em: 
<https://www.hse.ru/data/2019/03/04/1196348207/\%5BRachel_Kaplan,_Stephen_Ka plan\%5D_The_Experience_of_(b-ok.xyz).pdf>. Acesso em: 06 de dez. de 2021.

KELLERT, Stephen R. Nature By Design: the practice of biophilic design. Yale University Press, 2018.

LYNCH, Kevin. A imagem da cidade. Tradução Jefferson Luiz Camargo. 1a edição. São Paulo: Martins Fontes, 2006.

MCMICHAEL, Anthony. et al. In Comparative quantification of health risks: Global and regional burden of disease attributable to selected major risk factors, ed. Majid Ezzati, Alan D. Lopez, A. Rodgers, and C. Murray. Geneva: World Health Organization, $2004 . \quad$ Disponível em: <https://apps.who.int/iris/bitstream/handle/10665/42792/9241580348_eng_Volume1. pdf?sequence=1>. Acesso em: 06 de dez. de 2021.

ONU. ONU prevê que cidades abriguem 70\% da população mundial até 2050. Perspectiva Global Reportagens Humanas, 2019. Disponível em: <https://news.un.org/pt/story/2019/02/1660701>. Acesso em 23 de jul. de 2021.

PAIVA, Andréa. 12 princípios da Neuroarquitetura e do Neurourbanismo. NeuroAU, 2018. Disponível em: <https://www.neuroau.com/post/principios>. Acesso em : 22 de nov. de 2021.

PAIVA, Andréa. Emotions and senses: the relation between architecture, emotion and perception. Academy of Neuroscience for Architecture (ANFA), San Diego, 2018

PAIVA, Andréa. Neuroscience for architecture: how building design can influence behaviors and performance. Journal of Civil Engineering and Architecture, 2018. Disponível em: $<$ https://www.researchgate.net/publication/325016082_Neuroscience_for_Architectur 
e_How_Building_Design_Can_Influence_Behaviors_and_Performance>. Acesso em: 06 de dez. de 2021.

PAIVA, Andréa. 0 que o neurourbanismo nos ensina sobre as cidades. NeuroAU, 2019. Disponível em: <https://www.neuroau.com/post/o-que-oneurourbanismo-nos-ensina-sobre-nossas-cidades>. Acesso em : 27 de jul. de 2021.

RAMÓN Y CAJAL, Santiago. Advice For a Young Investigator. Traduzido por Neely Swanson e Larry W. Swanson. A Bradford Book, Cambridge, 2004.

SALINGAROS, Nikos. A. Biophilia \& healing environments healthy principles for designing the built world. Terrapin Bright Green, 2015. Disponível em: $<$ https://www.terrapinbrightgreen.com/wp-content/uploads/2015/10/Biophilia-HealingEnvironments-Salingaros-p.pdf>. Acesso em: 06 de dez. de 2021.

STIGSDOTTER, Ulrika. K. et al. Health promoting outdoor environments: Associations between green space, and health, health-related quality of life and stress based on a Danish national representative survey. National Library of Medicine, 2010. Disponível em <https://pubmed.ncbi.nlm.nih.gov/20413584/>. Acesso em: 06 de dez. de 2021.

SUNDQUIST, Kristina; GOLIN, Frank; SUNDQUIST, Jan. Urbanisation and incidence of psychosis and depression. National Library of Medicine. 2004. Disponível em: <https://pubmed.ncbi.nlm.nih.gov/15056572/>. Acesso 06 de dez. de 2021.

THOMPSON, Catharine Ward. et al. More green space is linked to less stress in deprived communities: Evidence from salivary cortisol patterns. Science Direct. Landscape and Urban Planning. Volume 105, Issue 3, 15 April 2012, Pages 221-229. Disponível em: $<$ https://www.sciencedirect.com/science/article/pii/S0169204611003665>. Acesso em: 06 de dez. de 2021. 
ULRICH, Roger S. Aesthetic and affective response to natural environment. Human Behavior Environmental Advanced Theory Research 6: 85-125, 1983. Disponível em: <https://www.researchgate.net/profile/Roger-Ulrich2/publication/232542780_Aesthetic_and_Affective_Response_to_Natural_Environme nt/links/53ecceba0cf26b9b7dbfe62b/Aesthetic-and-Affective-Response-to-NaturalEnvironment.pdf>. Acesso em: 06 de dez. de 2021.

WOLCH, Jennifer. et al. Childhood obesity and proximity to urban parks and recreational resources: A longitudinal cohort study. National Library of Medicine, 2010. Disponível em: <https://pubmed.ncbi.nlm.nih.gov/21075670/>. Acesso em: 06 de dez. de 2021.

Enviado: Novembro, 2021.

Aprovado: Janeiro, 2022. 